\section{Semiconducting Behavior in DNA Device Detected by Triple-Probe}

Deoxyribonucleic acid (DNA) is considered by some to be the ideal building block for nanoelectronic circuits, and, consequently, the electronic behavior of these molecules has attracted significant interest. It has been reported that the electrical characteristics of DNA can vary from those of an ohmic conductor to those of a widebandgap semiconductor. To better understand the carrier transport in DNA, a team of researchers at Fujixerox Co.'s Corporate Research Center in Kanagawa-ken, Japan, have developed a probing system for measuring the electrical properties of DNA molecules and other nanoscale samples.

As reported in the October 8 issue of Applied Physics Letters, H. Watanabe and coworkers have measured the electrical properties of a three-terminal single-molecule DNA device with a triple-probe atomic force microscope (T-AFM). A single DNA molecule was connected to the T-AFM with three carbon nanotube (CNT) electrodes, corresponding to a source, drain, and gate terminal. In addition to demonstrating the measurement capabilities of their T-AFM, the researchers showed that a salmon sperm DNA double strand was semiconducting.

The T-AFM system is comprised of a nanotweezers setup and a conventional AFM system equipped with a conductive, 8-nm diameter multiwalled CNT (MWCNT) probe. The nanotweezers, two 8-nm-diameter MWCNTs connected to a 100-nm-diameter glass needle, were used to position submicron DNA samples with 1-2-nm accuracy. A double strand of salmon sperm DNA was deposited on a $\mathrm{SiO}_{2} / \mathrm{Si}(100)$ substrate, and a singlewalled CNT (SWCNT) was deposited through the T-AFM adjacent to the DNA molecule to serve as the gate terminal. A three-dimensional piezo-actuator was used to position the nanotweezers such that the two CNT probes grabbed the salmon sperm DNA molecule and served as the source and drain terminals. To avoid the problems associated with electrostatic attraction of the probes both to each other and the substrate, the researchers employed a method whereby the CNT probes were continuously vibrated. When the sample was in the proper location, the vibrations were stopped and the nanotweezer probes were allowed to attain a fixed closed position and grab the sample. The MWCNT AFM probe was then used to apply a bias voltage to the SWCNT gate terminal.

The current-voltage $(I-V)$ curves, which were measured at a source-to-drain distance of $25 \mathrm{~nm}$ in room temperature dry nitrogen, were nonlinear, indicating semiconducting behavior. Furthermore, the voltage gap decreased with increasing gate bias voltage, indicating a conductance rise and demonstrating the switching characteristics of the gate-biased DNA molecule.

"While additional studies to further clarify carrier transport mechanisms in DNA are ongoing," said Watanabe, "we anticipate that DNA will play a major role in the future of advanced biosensors and molecular electronics."

STEFFEN K. KALDOR

\section{Lattice-Expanded $\mathrm{C}_{60}$ Achieves $T_{\mathrm{c}}$ of $117 \mathrm{~K}$}

Scientists J.H. Schön, Ch. Kloc, and B. Batlogg at Lucent Technologies/Bell Labs have shown that $C_{60}$ can act as a superconductor at temperatures as high as $117 \mathrm{~K}$, raising hopes for inexpensive current loss-free electronic devices based on organic compounds. Practical applications include quantum computers. The discovery is described in an article published on-line on August 31, 2001, by the journal Science on the Science Express Web site (www. sciencexpress.org), with the print version appearing in Science 293 (2001) p. 2432.

It is now understood that expanding the lattice of $\mathrm{C}_{60}$ increases the density of states at the Fermi level, leading to the increase of $T_{\mathrm{c}}$. The expanded lattice can be achieved by intercalation of trihalomethane $\mathrm{CHX}_{3}$ with $\mathrm{C}_{60}$ crystals. The researchers intercalated $\mathrm{C}_{60}$ single crystals with $\mathrm{CHCl}_{3}$ and $\mathrm{CHBr}_{3}$ in a solution to form co-crystals. The lattice parameter increased from $14.16 \AA$ for undoped $\mathrm{C}_{60}$ to $14.28 \AA$ and $14.43 \AA$ for $\mathrm{C}_{60} / \mathrm{CHCl}_{3}$ and $\mathrm{C}_{60} / \mathrm{CHBr}_{3}$, respectively. Field-effect devices were created and researchers induced high densities of electrons or holes by gate doping. Since the hole-phonon interaction seems to be stronger than the electron-phonon interaction and the density of states in the valence band is higher than in the conduction band, the $T_{\mathrm{c}}$ for hole doping is higher than for electron doping. When the hole density reached approximately one hole per molecule, superconductivity above $1.7 \mathrm{~K}$ was observed. More holes led to higher values of $T_{\mathrm{c}}$, and the value of $T_{c}$ depended on the size of the intercalant molecule and the hole concentration. The highest $T_{\mathrm{c}}$ of $117 \mathrm{~K}$ was observed in $\mathrm{C}_{60} / \mathrm{CHBr}_{3}$ with 3-3.5 holes per $\mathrm{C}_{60}$ molecule. The research team also found that the increase of $T_{\mathrm{c}}$ with increased spacing between the $\mathrm{C}_{60}$ molecules follows the general trend of alkali-metal-doped $\mathrm{C}_{60}$, which is explained in terms of the increased density of states at the Fermi level. The team suggests that if the lattice parameter can be further increased by $\sim 1 \%$, a $T_{\mathrm{c}}$ exceeding $150 \mathrm{~K}$ may result.

The only other known superconductors that work at this and higher temperatures are copper oxide superconductors. These, however, have other problems. The physics that governs copper oxide superconductors is nonconventional and not well understood, and they are highly anisotropic and granular, which makes it difficult to fabricate useful devices. The $\mathrm{C}_{60}$ superconductors, on the other hand, according to the researchers, are less expensive, the physics is better understood since they seem to act as conventional superconductors, and they have a potential for practical devices.

SHIMING Wu

\section{Superconducting Quantum Interference Device (SQUID) on} $\mathrm{MgB}_{2}$ Thin Films Close to a Reality

Researchers from the University of Twente in the Netherlands have fabricated a superconducting quantum interference device (SQUID) circuit based on thin films of $\mathrm{MgB}_{2}$, a newly discovered non-copper oxide superconductor with a critical transition temperature $\left(T_{c}\right)$ of $39 \mathrm{~K}$. The advantages of this material for electronic applications include the large charge-carrier density, isotropy of the material, and strong links at grain boundaries. The technology to fabricate Josephson junctions in these high-quality films is essential for practical circuits.

Because of the post-annealing step involved in preparing the $\mathrm{MgB}_{2}$ films, the conventional tri-layer Josephson junctions could not be easily constructed. Fortunately, the weak links in a SQUID circuit can also be realized on a single-layer film by creating nanobridges in a superconducting ring.

As described in the October 1 issue of Applied Physics Letters, the researchers deposited thin films of $200-\mathrm{nm} \mathrm{MgB}_{2}$ on $\mathrm{MgO}$ substrates by pulsed laser ablation in a two-step in situ process. Standard photolithography and argon ion-beam milling were used to pattern the SQUID ring and contact paths on the film. The structure contained two striplines into which nanobridges were structured by direct focusedion-beam (FIB) milling. The dimensions of the nanobridges were $70 \mathrm{~nm}$ wide by $150 \mathrm{~nm}$ long and $150 \mathrm{~nm}$ high. The $T_{\mathrm{c}}$ of the structure was found to be $22 \mathrm{~K}$.

The critical current of the SQUID at $T=$ $4.2 \mathrm{~K}$ is $1.5 \mathrm{~mA}$, which corresponds to a critical current density of $7 \times 10^{6} \mathrm{~A} / \mathrm{cm}^{2}$. At $10 \mathrm{~K}$, a modulation voltage of $30 \mu \mathrm{V}$ was observed. Voltage modulation was observed up to $20 \mathrm{~K}$. The structures are very stable over time and are insensitive to thermal cycling or exposure to moisture. 